\title{
Personality traits distinguishing dementia with Lewy bodies from Alzheimer disease
}

James E. Galvin, MD, $\mathrm{MPH}$

Heather Malcom David Johnson, PhD John C. Morris, MD

Address correspondence and reprint requests to Dr. James E. Galvin, Alzheimer Disease Research Center, Washington University School of Medicine, 4488 Forest Park, Suite 130, St. Louis, MO 63108 galvinj@neuro.wustl.edu
ABSTRACT Objective: To identify personality traits that distinguish dementia with Lewy bodies (DLB) from Alzheimer disease (AD). Methods: We examined 290 participants enrolled in a longitudinal study (nondemented control $=34, \mathrm{DLB}=128, \mathrm{AD}=128$ ) followed to autopsy. As part of the annual interview with the collateral source, the clinician asked about specific changes in personality, interests, and drives based on items from the Blessed Dementia Scale (BDS). Statistical analysis was performed using $\chi^{2}$ and Fisher exact tests. Factor analysis was performed to determine underlying structure and receiver operating characteristic curves assessed the ability for each of three derived factors to discriminate DLB from AD. Results: The sample was evaluated for a mean of 4.8 visits (range 1 to 14 ) with a mean age of $77.6 \pm 9.9$ years. The participants' cognitive status ranged from nondemented (Clinical Dementia Rating [CDR] 0) through all stages of dementia (CDR $\geq 0.5$ ). Personality traits that distinguished DLB included diminished emotional responsiveness $(p=0.004)$, relinquishing hobbies $(p=0.01)$, growing apathy $(p=0.03)$, and purposeless hyperactivity $(p=0.003)$. Factor analyses of the BDS revealed a PASSIVE factor (diminished emotional responsiveness, relinquished hobbies, growing apathy, and purposeless hyperactivity) explaining 10.4\% of variance and that DLB was more likely to manifest these personality traits than AD ( $p=0.001)$. The PASSIVE factor discriminated DLB from $\mathrm{AD}$ (area under the curve $=0.61,95 \% \mathrm{Cl}: 0.54$ to $0.68, p=0.006$ ). Any change in personality is associated with the presence of visual hallucinations. Conclusions: Our results suggest that incorporating a brief, simple inventory of personality traits may improve the identification of individuals with dementia with Lewy bodies. NEUROLOGY 2007;68:1895-1901

Identifying the earliest features of dementia enables clinicians to implement therapy as soon as possible and provides patients and families more time to plan for the accompanying progressive decline. Much effort has focused on the cognitive symptoms of dementia syndromes such as impairments in memory, visuospatial, attention, and executive function. An alternative approach to improve early detection is to study noncognitive symptoms such as behavioral and personality changes that may precede diagnosis or occur early in the course of the dementia. ${ }^{1-3}$

Alzheimer disease (AD) is the most common form of dementia. While memory impairment is a common and early cognitive sign of disease onset, $\mathrm{AD}$ may be heralded by changes in $\operatorname{mood}^{4,5}$ and personality. ${ }^{3}$ Although a number of studies have used longitudinal designs to examine personality changes after clinical diagnosis, ${ }^{6-8}$ few have evaluated individuals prior to dementia onset to determine which personality traits precede the onset of AD. One prospective study reported that personality changes in undiagnosed people predicted dementia with 2 years follow-up. ${ }^{9}$ Another recent study evaluated nondemented and demented individuals followed longitudinally, some to autopsy. ${ }^{3}$ Personality traits described for AD include neuroticism, ${ }^{10}$ decreased conscientiousness, ${ }^{11}$ increased rigidity and egocentricity, ${ }^{8}$ and coarsening of affect, ${ }^{12}$ although most studies rely on retrospective design to track these changes, a method limited by systematic biases in personality perception ${ }^{13}$ and recall bias.

Dementia with Lewy bodies (DLB) is the second most common neurodegenerative cause of

From the Alzheimer Disease Research Center (J.E.G., H.M., D.J., J.C.M.) and Departments of Neurology (J.E.G., D.J., J.C.M.), Anatomy and Neurobiology (J.E.G.), and Pathology and Immunology (J.C.M.), Washington University School of Medicine, St. Louis, MO.

Supported by grants from the National Institute on Aging (K08 AG20764, P01 AG03991, and P50 AG05681), the American Federation for Aging Research, and a generous gift from the Alan A and Edith L Wolff Charitable Trust. Dr. Galvin is a recipient of the Paul Beeson Physician Faculty Scholar Award in Aging Research.

Disclosure: The authors report no conflicts of interest. 
dementia, characterized by extrapyramidal signs (EPS), visual hallucinations, and cognitive fluctuations. ${ }^{14,15}$ There is a paucity of data available evaluating the personality characteristics of DLB, or whether such changes may be used to enhance accurate clinical diagnosis. We analyzed data from a longitudinal study that included annual assessments of personality from demented individuals as well as nondemented individuals, some of who eventually developed AD or DLB. Individuals were followed to autopsy with pathologic confirmation of underlying dementia etiologies. If personality changes are early features of dementia syndromes, perhaps even before the emergence of detectable cognitive impairments, then personality changes may assist clinicians in disease detection and differentiation in conjunction with other DLB features.

METHODS Research participants. Beginning in 1979, over 3,000 individuals have been enrolled in our longitudinal studies of healthy aging and dementia and over 800 of these participants have been studied postmortem. ${ }^{16-18}$ Nondemented and demented individuals undergo identical annual assessments. For this study, we examined the clinical records of 290 participants (nondemented control $=34, \mathrm{DLB}=128, \mathrm{AD}=$ 128) followed longitudinally. Because the diagnosis of DLB has evolved over the course of the longitudinal study, we used the neuropathologically defined groups for comparison rather than the clinically defined where the majority of the cases prior to 2000 were diagnosed with dementia of the Alzheimer type. All cases were reviewed independently by two authors (J.E.G., D.J.K.). All normal and DLB cases that came to autopsy were selected. AD cases were selected from all autopsies performed after the advent of alpha-synuclein staining (circa 2000) to detect the presence of Lewy bodies, matched to the DLB group for age, dementia severity, and duration of follow-up. The Washington University Human Studies Committee approved all procedures.

Clinical data. Experienced clinicians (neurologists, psychiatrists, and geriatricians) conducted independent semistructured interviews with the participant and a knowledgeable collateral source (usually the spouse or close family member) to capture features suggestive of a dementing disorder. ${ }^{18,19}$ The clinical assessment protocol included evaluation of performance in activities of daily living, an aphasia battery, construction tasks, a health and medication history to assess for comorbid disease, and a detailed neurologic examination. The Clinical Dementia Rating (CDR) was used to determine the presence or absence of dementia and to stage its severity from nondemented (CDR 0) to severe dementia (CDR 3). ${ }^{20}$ The sum of the individual category ratings (sum of boxes or $\mathrm{SB}$ ) provides a quantitative expansion of the CDR and ranges from 0 (no impairment) to 18 (maximum impairment). ${ }^{17}$ In our sample, the CDR 0.5 rating equates with very mild dementia ${ }^{21}$ and is the threshold to distinguish nondemented from demented status. In other samples
CDR 0.5 has been used as the threshold for the diagnosis of mild cognitive impairment (MCI). ${ }^{22}$ In both cases the CDR is useful to detect the change in cognitive abilities from a prior level of function and also to assess interference with accustomed activities. The validity has been established by correlation with neuropathologic features observed at autopsy. ${ }^{18}$

Information on the presence of EPS including rest tremor, bradykinesia, postural instability, and cogwheel rigidity was coded as present or absent. Abnormalities of gait were also collected as present or absent. There are no standardized measures of cognitive fluctuation; however, over the course of the longitudinal project, we have collected information regarding fluctuations in onset and course of cognitive impairment. These data were coded as present or absent.

Personality and behavioral data. As part of the annual interview with the collateral source, the clinician asked the informant about specific changes in personality, interests, and drives based on items from the Blessed Dementia Scale (BDS) ${ }^{23}$ : increased rigidity, increased egocentricity, loss of concern for feelings of others, coarsening of affect, impaired emotional control, hilarity in inappropriate situations, diminished emotional responsiveness, sexual misdemeanor appearing in old age, relinquished hobbies, diminished initiative or growing apathy, and purposeless hyperactivity. Each item was scored by the clinician as present (yes; that change had occurred) or absent (no; that change had not occurred). Additionally, at each assessment, the informant was queried as to presence or absence of psychotic features: paranoid or persecutory delusions, and auditory or visual hallucinations.

Neuropathology. All brains were examined with a standard protocol. ${ }^{24}$ Following fixation in neutral buffered $10 \%$ formalin, tissue blocks were taken from 30 brain regions. Sections $(6$ $\mu \mathrm{m})$ from paraffin-embedded tissue blocks were stained with hematoxylin-eosin, Gallyas and modified Bielschowsky silver stains, and immunohistochemical methods. ${ }^{24}$ Histologic criteria for AD were based on several staging paradigms. Quantification of diffuse and neuritic amyloid deposition were calculated in five cortical regions with $10 \mathrm{~mm}^{2}$ microscopic fields in each region. ${ }^{24}$ Braak neurofibrillary scores were determined, ${ }^{25}$ and the National Institute on Aging (NIA)-Reagan ${ }^{26}$ neuropathologic probability estimates of $\mathrm{AD}$ were performed taking into account the distribution of neuritic plaques and neurofibrillary tangles. DLB pathologic diagnoses were made according to published criteria. ${ }^{14}$ Cases were screened for Lewy bodies with antibodies to alpha-synuclein using a semiquantitative grading of severity of Lewy-related pathology into none, mild, moderate, severe, and very severe. ${ }^{14}$ Sections were also examined for the presence of cortical and subcortical infarcts and hemorrhages. None of the cases used in these analyses had substantial vascular burden that was thought to contribute to dementia.

Statistical analysis. Analyses were performed using SPSS v13.0 statistical software (SPSS Inc., Chicago, IL). Change from nondemented status (global CDR 0) to dementia (global CDR $\geq 0.5$ ) was the primary outcome measure. The groups were compared using $t$ tests and analysis of variance (ANOVA) for quantitative variables and $\chi^{2}$ tests of independence for categorical variables. The presence of clinical features associated with dementia was collected as dichotomous variables $(1=$ symptom present, $0=$ symptom absent) at baseline and at any time during annual assessments.

The frequency of these features was compared with $\chi^{2}$ and Fisher exact tests. Three cross-sectional moments were consid- 
Table $1 \quad$ Sample characteristics

$\begin{array}{lllll}\text { Variable } & \text { Nondemented, } \mathrm{n}=34 & \mathrm{AD}, \mathrm{n}=128 & \mathrm{DLB}, \mathrm{n}=128 & \mathrm{p} \text { Value } \\ \text { Age at first visit, } \mathrm{y} & 80.6(9.9) & 78.1(10.3) & 76.4(9.3) & \mathrm{NS} \\ \text { Age at diagnosis, } \mathrm{y} & 84.4(6.7) & 81.8(10.9) & 80.8(10.4) & \mathrm{NS} \\ \text { Age at death, } \mathrm{y} & 91.9(7.7) & 84.4(10.1) & 81.7(9.4) & 0.01 \\ \text { \% Men } & 52.9 & 44.4 & 50.4 & \mathrm{NS} \\ \text { Education, } \mathrm{y} & 13.2(4.2) & 13.8(3.5) & 12.9(3.5) & \mathrm{NS} \\ \text { Number of assessments } & 6.5(7.0) & 4.9(3.9) & 4.2(3.4) & \mathrm{NS} \\ \text { CDR at 1st visit } & 0.07(0.18) & 0.56(0.47) & 0.85(0.60) & <0.001 \\ \text { CDR-SB at 1st visit } & 0.33(0.85) & 3.0(2.9) & 4.7(3.7) & <0.001 \\ \text { CDR at diagnosis } & 0.31(0.26) & 0.59(0.41) & 0.79(0.45) & <0.001 \\ \text { CDR-SB at diagnosis } & 1.2(1.3) & 3.2(2.7) & 4.5(3.0) & <0.001 \\ \text { Extrapyramidal signs, \% } & 11.1 & 20.8 & 49.2 & <0.001 \\ \text { Visual hallucinations, \% } & 2.9 & 13.3 & 32.0 & <0.001 \\ \text { Fluctuations, \% } & 8.3 & 8.3 & 17.9 & \mathrm{NS}\end{array}$

Values are means (SD). For nondemented group, age at diagnosis is taken at cross-sectional moment equal to mean number of visits for demented (AD, DLB) groups.

$\mathrm{AD}=$ Alzheimer disease; $\mathrm{DLB}=$ dementia with Lewy bodies; CDR = Clinical Dementia Rating; CDR-SB = CDR sum of boxes

ered for the analyses. First, features were compared at the time of the first clinical assessment, regardless of dementia status. Second, features were compared at the time of dementia diagnosis, corresponding to the time of the first $\mathrm{CDR} \geq 0.5$ (i.e., be demented). Third, the features were compared if they were ever present during the course of longitudinal follow-up.

Factor analysis (Varimax rotation) was completed on the BDS personality traits to determine underlying structure. Factor extraction was based on both the Kaiser-Guttman rule of retaining components with eigenvalues greater than one and inspection of scree plots of eigenvalues vs their ordinal positions. Although the sample size does not satisfy more conservative rules of factor analysis, ${ }^{27,28}$ this sample size satisfies the subject to variable ratio $(10: 1)$ rule of thumb, ${ }^{29}$ which has been supported by Monte Carlo studies findings, indicating these ratios are sufficiently powerful to establish preliminary clinical results when the total sample size is greater than $100 . .^{30}$ Three factors were identified and factor scores were calculated. Between-group comparisons of quantitative measures were conducted using ANOVA. Receiver operator characteristic (ROC) curves and the area under the ROC curve (AUC) were generated to reflect graphically and quantitatively the ability of each of the three factor scores to discriminate between $\mathrm{AD}$ and DLB. Pearson correlations were used to test the strength of association between the DLB clinical criteria (EPS, visual hallucinations, fluctuation) and the presence of personality traits.

RESULTS Sample characteristics. The mean age ( \pm SD) of the sample was $77.6 \pm 9.9$ years with $13.3 \pm 3.6$ years of education. The sample ethnicity was $93 \%$ white and $52 \%$ were men. The sample was evaluated over a mean of 4.8 visits (range 1 to 14). The participants' cognitive status ranged from nondemented (CDR 0) through all stages of dementia $(\mathrm{CDR} \geq 0.5)$. Demographic categories by group are shown in table 1 . Visual hallucinations $(p<0.001)$ and EPS $(p<0.001)$ were more frequently present in DLB than AD or nondemented adults (table 1). Fluctuations were more frequently present in DLB but did not reach significance $(p=0.056)$.

Comparison of personality and behavioral characteristics between groups. Nondemented vs demented at first clinical assessment. Demented individuals (AD or DLB) were more likely to exhibit increased rigidity $(p=0.01)$, to exhibit impaired emotional control $(p=0.03)$, to exhibit diminished emotional responsiveness $(p=0.04)$, to have relinquished hobbies $(p=0.003)$, and to have diminished initiative $(p<$ 0.001 ) (table 2). This suggests that the presence of these personality features at the first office visit may give clues to the presence of dementia.

Nondemented vs demented everpresent during longitudinal follow-up. Demented individuals (AD or DLB) displayed many personality and behavioral changes during the progression of disease compared with individuals who remained nondemented and were found to have no dementia pathology at autopsy. Two personality traits from the BDS showed no difference between nondemented individuals and those with AD or DLB: inappropriate hilarity $(p=0.24)$ and sexual misdemeanor $(p=0.29)$. This suggests that these personality traits are not prominent features of $\mathrm{AD}$ or DLB.

DLB vs AD. DLB vs AD at first clinical assessment. Personality and behavioral traits that were more frequent in those individuals who had neuropathologically confirmed DLB compared with those with 


\begin{tabular}{|c|c|c|c|c|c|c|c|c|c|c|c|c|}
\hline \multirow{3}{*}{$\begin{array}{l}\text { Table } 2 \\
\text { Variable (\%) }\end{array}$} & oran & litur & behav & trait & by dias & nostic & ator & & & & & \\
\hline & \multicolumn{4}{|c|}{ At first visit } & \multicolumn{4}{|c|}{ At dementia diagnosis } & \multicolumn{4}{|c|}{ Ever present } \\
\hline & ND & $A D$ & DLB & $p$ Value & ND & $A D$ & DLB & $p$ Value & ND & $A D$ & DLB & $p$ Value \\
\hline Increased rigidity & 6.7 & 24.1 & 27.0 & 0.06 & 12.5 & 26.1 & 27.5 & 0.64 & 23.5 & 49.3 & 54.7 & 0.005 \\
\hline Increased egocentricity & 10.0 & 12.1 & 23.8 & 0.03 & 12.5 & 11.9 & 25.0 & 0.03 & 23.5 & 37.5 & 44.5 & 0.07 \\
\hline Loss of concern & 3.3 & 9.8 & 23.0 & 0.002 & 0 & 10.1 & 23.3 & 0.009 & 11.8 & 32.4 & 43.8 & 0.002 \\
\hline Coarsening of affect & 6.7 & 10.5 & 7.9 & 0.69 & 12.5 & 11.8 & 8.3 & 0.66 & 11.8 & 26.5 & 30.5 & 0.09 \\
\hline Impaired emotional control & 3.3 & 19.5 & 18.3 & 0.09 & 0 & 21.0 & 18.3 & 0.33 & 14.7 & 37.5 & 47.7 & 0.002 \\
\hline Inappropriate hilarity & 3.3 & 3.8 & 7.1 & 0.43 & 12.5 & 4.3 & 6.7 & 0.51 & 5.9 & 11.8 & 14.1 & 0.42 \\
\hline $\begin{array}{l}\text { Diminished emotional } \\
\text { responsiveness }\end{array}$ & 3.3 & 12.2 & 23.8 & 0.005 & 0 & 13.7 & 23.3 & 0.06 & 8.8 & 33.1 & 50.8 & $<0.001$ \\
\hline Sexual misdemeanor & 0 & 2.3 & 5.6 & 0.19 & 0 & 2.6 & 5.1 & 0.51 & 2.9 & 7.4 & 8.6 & 0.53 \\
\hline Hobbies relinquished & 6.7 & 26.5 & 39.7 & 0.001 & 12.5 & 29.7 & 38.3 & 0.16 & 17.6 & 55.1 & 70.3 & $<0.001$ \\
\hline Growing apathy & 6.7 & 31.8 & 46.4 & $<0.001$ & 12.5 & 35.6 & 47.1 & 0.05 & 17.6 & 59.6 & 72.7 & $<0.001$ \\
\hline Purposeless hyperactivity & 6.7 & 6.9 & 17.5 & 0.02 & 12.5 & 7.7 & 17.5 & 0.08 & 8.8 & 27.2 & 44.5 & $<0.001$ \\
\hline Paranoia & 0 & 8.9 & 14.3 & 0.12 & 0 & 8.9 & 14.8 & 0.25 & 0 & 24.3 & 29.7 & 0.001 \\
\hline Auditory hallucinations & 0 & 0.8 & 7.1 & 0.02 & 0 & 0 & 7.4 & 0.01 & 0 & 8.1 & 18.0 & 0.003 \\
\hline Visual hallucinations & 5.3 & 2.4 & 16.8 & $<0.001$ & 14.3 & 1.8 & 16.7 & 0.001 & 2.9 & 14.0 & 32.0 & $<0.001$ \\
\hline
\end{tabular}

$\mathrm{ND}=$ nondemented $\mathrm{AD}=$ Alzheimer disease; $\mathrm{DLB}=$ dementia with Lewy bodies

$\mathrm{AD}$ included increased egocentricity $(p=0.01)$, loss of concern for others $(p=0.004)$, diminished emotional responsiveness $(p=0.02$ ), relinquished hobbies $(p=0.02)$, growing apathy $(p=0.02)$, purposeless hyperactivity $(p=0.009)$, and the presence of auditory $(p=0.01)$ and visual hallucinations $(p<0.001)$. These features may provide clues to support DLB diagnosis at first encounter with the patient.

DLB vs AD at time of dementia diagnosis/onset. The nature of our longitudinal study allows us to detect the onset of dementia in previously nondemented individuals we have been following. At the time of the first non-0 CDR, the DLB group was more likely to exhibit increased egocentricity $(p=0.009)$, loss of concern for others $(p=0.006)$, and purposeless hyperactivity $(p=0.02)$. Auditory $(p=0.003)$ and visual $(p<0.001)$ hallucinations were also more common in DLB.

$D L B$ vs $A D$ ever present during longitudinal follow-up. Over the course of longitudinal follow-up, from mild stages of dementia (CDR 0.5) until terminal stages (CDR 3), many personality traits were common between AD and DLB. Personality traits that appeared to distinguish DLB included diminished emotional responsiveness $(p=0.004)$, relinquishing hobbies $(p=0.01)$, growing apathy $(p=0.03)$, and purposeless hyperactivity $(p=0.003)$. Behavioral features included the presence of auditory $(p=$ $0.02)$ and visual $(p<0.001)$ hallucinations. There were no differences in the presence of paranoid or persecutory delusions between AD and DLB.
Discriminating DLB from AD. Principal components analysis was performed on personality traits from the BDS if ever present during longitudinal followup. Factor analyses confirmed a three-factor solution. Factor 1 (termed IRRITABLE), which includes increased rigidity, egocentricity, loss of concern, and coarsening affect and impaired emotional control, explains $32.8 \%$ of the variance. Factor 2 (termed PASSIVE), which includes diminished emotional responsiveness, relinquished hobbies, growing apathy, and purposeless hyperactivity, explains $12.2 \%$ of variance. Factor 3 (termed DISINHIBITED) includes inappropriate hilarity and sexual misdemeanor and explains an additional $10.4 \%$ of the variance. Factor loadings, rotated eigenvalues, and variances are reported in table 3. The factor analyses were repeated for the other two crosssectional moments (at first visit and at dementia diagnosis) and the results were identical.

ANOVA indicated group differences on Factor 2 (PASSIVE) $[\mathrm{F}(1,254)=10.5, p=0.001]$, indicating that DLB was more likely to manifest these personality traits than $\mathrm{AD}$. ROC curves were generated to measure the effectiveness of each of the three factors in classifying DLB vs AD (figure). The PASSIVE factor discriminated DLB from AD (AUC $=0.61,95 \%$ CI: 0.54 to $0.68, p=0.003)$. The IRRITABLE factor did not discriminate between DLB and AD (AUC = 0.52 , $95 \%$ CI: 0.45 to 0.59 ) and may instead represent a general dementia personality phenotype. The DISINHIBITED factor also did not discriminate 


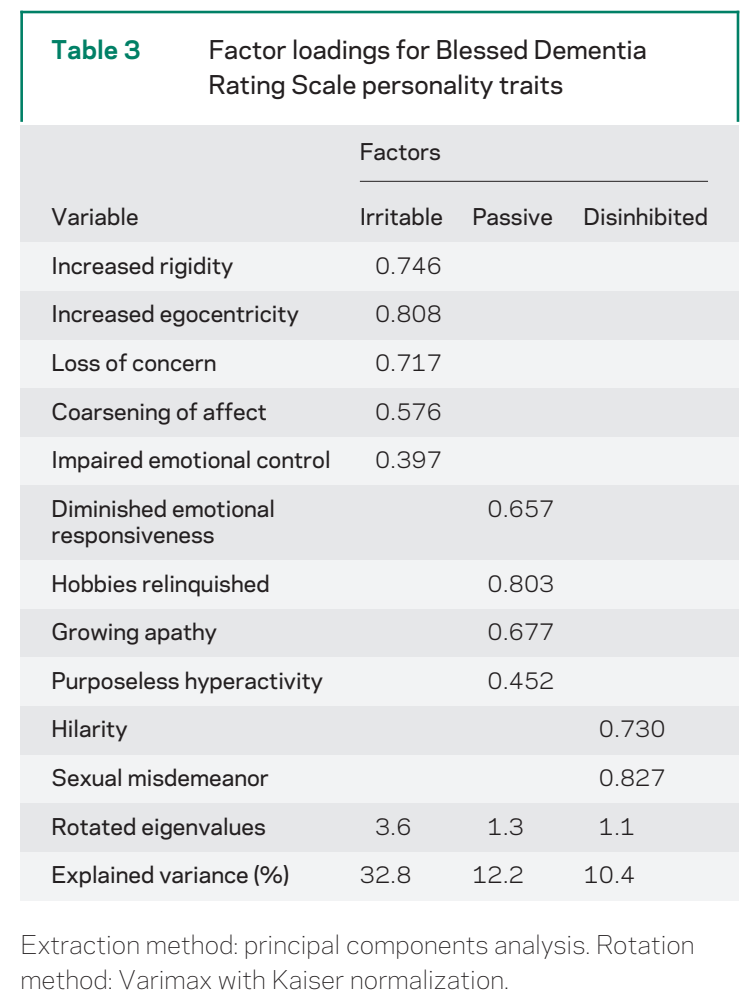

DLB from $\mathrm{AD}$ ( $\mathrm{AUC}=0.52,95 \% \mathrm{CI}: 0.45$ to 0.59 ) and was uncommon in either AD or DLB.

Correlation between DLB clinical features and personality changes. Correlation analyses were performed examining the relationship between the DLB consensus features of EPS, visual hallucinations, and fluctuations with each of the personality factors (IRRITABLE, PASSIVE, DISINHIBITED) in the $\mathrm{AD}$ and DLB cases (table 4). Any change in personality correlated strongly with the presence of visual hallucinations. The PASSIVE traits had the stron-

The receiver operator characteristic (ROC) curve comparing the factor scores of dementia with Lewy bodies (DLB) and Alzheimer disease (AD). Three factors were identified. The area under the ROC curve for the PASSIVE factor $=0.61$ (95\% Cl: 0.54 to $0.68, p=$ 0.003), suggesting good ability to discriminate between DLB and AD. The other two factors IRRITABLE and DISINHIBITED did not discriminate between DLB and AD. See text for details of personality traits included in each factor. gest correlation with hallucinations $(\mathrm{r}=0.24, p<$ 0.001 ). The presence of fluctuations correlated with IRRITABLE traits $(r=0.23, p=0.002)$. The presence of EPS did not correlate with any of the personality changes. The three personality factors did not correlate with each other. These relationships also held true when only the DLB group was considered.

DISCUSSION We found that personality changes in DLB included diminished emotional responsiveness, relinquishing hobbies, growing apathy, and purposeless hyperactivity, often accompanied by the presence of auditory and visual hallucinations. Personality changes in general and passive traits in particular correlated with the presence of hallucinations but not with parkinsonism. Fluctuations were more commonly associated with irritability. These personality changes may assist in identifying individuals with DLB, and help discriminate DLB from AD.

We also found that compared with nondemented older adults there is a general dementia phenotype of increased rigidity, increased egocentricity, loss of concern for the feelings of others, coarsening of affect, and impaired emotional control. These findings are consistent with other studies reporting personality changes in $\mathrm{AD} \cdot{ }^{6-8,12,31}$ In the absence of autopsy confirmation, however, it is impossible to know how many individuals in these prior studies of AD personality traits had concurrent Lewy bodies.

As a complementary strategy to using the presence of cognitive impairments to improve detection of dementia, studying noncognitive symptoms such as behavioral and personality changes that can precede the onset of cognitive symptoms by several years ${ }^{5}$ may provide opportunities to make diagnoses early in the course of the dementia. ${ }^{1-3}$ This avenue of research may eventually address how to discriminate between different types of dementia on the basis of early personality change. Early, unique personality change associated with different types of dementia may improve differential diagnosis. Personality change has been used to differentiate frontotemporal dementia from $\mathrm{AD}$ at later stages of the disease ${ }^{32,33}$ and to differentiate among head injury, stroke, and AD. ${ }^{34}$ Reports of personality change in DLB, however, are lacking.

Our study has limitations. Ratings provided from the BDS, although easily administered, are coarse and non-quantifiable. Informants indicate whether change is present or absent; however, there is no consideration of the degree of change. A structured and more sophisticated interview such as the Neuroticism Extroversion Openness-Personality Inventory (NEO-PI), ${ }^{35-37}$ Neuropsychiatric Invento- 


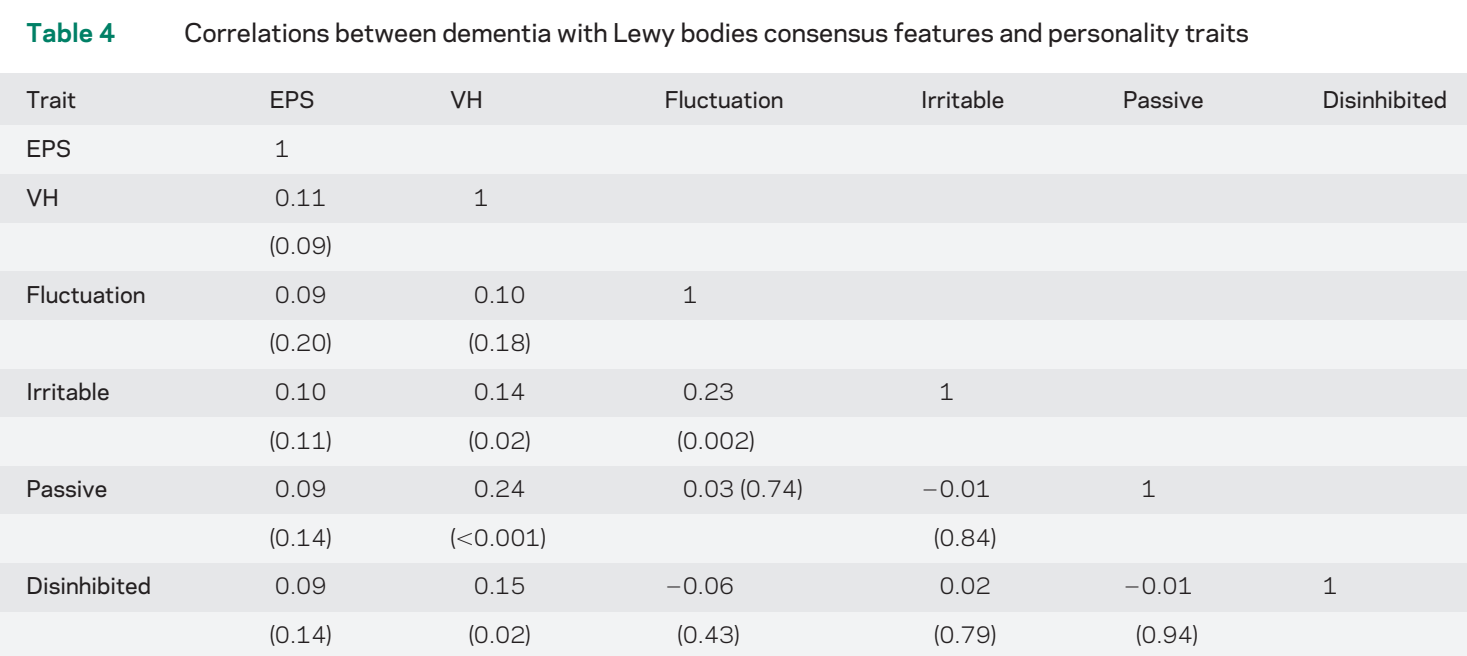

Values are presented as correlation coefficient ( $p$ value). EPS $=$ extrapyramidal signs; $\mathrm{VH}=$ visual hallucinations.

ry ${ }^{38,39}$ or the BEHAVE-AD ${ }^{40}$ might yield more detailed and comprehensive information regarding what facets of personality change first and what degree of change is indicative of dementia. The sample was not population-based, and thus we cannot directly compare our findings with epidemiologic data. Individuals who came to autopsy may differ from those who did not; however, this sample is representative of our larger study of over 3,000 individuals. As with any volunteer sample, there may be selection biases, thus limiting generalization of the results. Our sample is largely white, so it is unknown how these results generalize to other ethnicities. Our convenience sample has demographic attributes and comorbid disorders that reflect those of the similarly aged population in the greater St. Louis metropolitan area. ${ }^{18}$ It is difficult to accurately capture cognitive fluctuation. Fluctuations may represent brief interruptions of consciousness, periods of increased confusion and cognitive impairment, episodes of diminished arousal, or what seem to be periods of prolonged sleep. Rating scales have been developed to help assess these fluctuations but many issues are unresolved. ${ }^{41}$ We therefore used dichotomous variables to capture informant reports of fluctuating onset and course of dementia. Finally, nearly all DLB cases that came to autopsy had sufficient numbers of senile plaques and neurofibrillary tangles to also meet pathologic criteria for $\mathrm{AD},{ }^{26}$ therefore it was impossible to separate the influence of AD pathology on the DLB personality phenotype. However, our factor analyses suggest increased passivity and purposeless, repetitive behaviors characterize those individuals with DLB.

The unique aspects of this study relate to the large number of individuals followed longitudinally over a long surveillance period (mean 4.8 years) with prospective collection of personality and behavioral data, and autopsy verification of neuropathology. In most office settings, standardized scales such as the NEO-PI ${ }^{35}$ are not performed due to length of time and lack of training. This study examined whether the presence of personality and behavioral features which can be collected during a routine office visit corresponds with the diagnosis of DLB and correlates with clinical features. Our results suggest that incorporating a brief, simple inventory of personality traits such as the contained within the $\mathrm{BDS}^{23}$ can assist in the identification of individuals with DLB.

\section{ACKNOWLEDGMENT}

The authors thank the Clinical and Neuropathology of the AD Research Center for their expert clinical and neuropathologic characterizations.

Received September 10, 2006. Accepted in final form January 30, 2007.

\section{REFERENCES}

1. Oppenheim G. The earliest signs of Alzheimer's disease. J Geriatr Psychiatry Neurol 1994;7:116-120.

2. Wild KV, Kaye JA, Oken BS. Early noncognitive change in Alzheimer's disease and healthy aging. J Geriatr Psychiatry Neurol 1994;7:199-205.

3. Balsis S, Carpenter BD, Storandt M. Personality change preceded clinical diagnosis of dementia of the Alzheimer type. J Gerontol B Psychol Sci Soc Sci 2005;60:98-101.

4. Bartolini M, Coccia M, Luzzi S, Provincialli L, Cervolo MG. Motivational symptoms of depression may mask preclinical Alzheimer's disease in elderly subjects. Dement Geriatr Cogn Disord 2005;19:31-36.

5. Jost BC, Grossberg GT. The evolution of psychiatric symptoms in Alzheimer's disease: a natural history study. J Am Geriatr Soc 1996;44:1078-1081.

6. Petry S, Cummings JL, Hill MA, Shapira J. Personality alterations in dementia of the Alzheimer type. Arch Neurol 1988;45:1187-1190. 
7. Petry S, Cummings JL, Hill MA, Shapira J. Personality alterations in dementia of the Alzheimer type: A threeyear follow-up study. J Geriatr Psychiatry Neurol 1989;2: 203-207.

8. Rubin EH, Morris JC, Berg L. The progression of personality changes in senile dementia of the Alzheimer's type. J Am Geriatr Soc 1987;35:721-725.

9. Smith-Gamble V, Baiyewu O, Perkins AJ, et al. Informant reports of changes in personality predict dementia in a population-based study of elderly African Americans and Yoruba. Am J Geriatr Psychiatry 2002;10:724-732.

10. Siegler IC, Dawson DV, Welsh KA. Caregiver ratings of personality change in Alzheimer's disease patients: a replication. Psychol Aging 1994;9:464-466.

11. Strauss ME, Pasupathi M, Chatterjee A.. Concordance between observers in descriptions of personality change in Alzheimer's disease. Psychol Aging 1993;8:,475-480.

12. Bozzola FG, Gorelick PB, Freels S. Personality changes in Alzheimer's disease. Arch Neurol 1992;49:297-300.

13. Strauss ME, Stuckey JC, Pasupathi M, Moore A. Accuracy of retrospective descriptions of personality during the course of Alzheimer's disease. J Clin Geropsychology 1997;3:93-99.

14. McKeith IG. Consensus guidelines for the clinical and pathologic diagnosis of dementia with Lewy bodies (DLB): Report of the Consortium on DLB International Workshop. J Alzheimer Dis 2006;9(3 Suppl):417-423.

15. Galvin JE. Dementia with Lewy bodies. Arch Neurol 2003;60:1332-1335.

16. Berg L, Hughes CP, Coben LA, Danziger WL, Martin RL, Knesevich J. Mild senile dementia of Alzheimer type: research diagnostic criteria, recruitment, and description of a study population. J Neurol Neurosurg Psychiatry 1982; 45:962-968.

17. Berg L, Miller JP, Baty J, Rubin EH, Morris JC, Figiel G. Mild senile dementia of the Alzheimer type. 4. Evaluation of intervention. Ann Neurol 1992;31:242-249.

18. Galvin JE, Powlishta KK, Wilkins K, et al. Predictors of preclinical Alzheimer disease and dementia: a clinicopathologic study. Arch Neurol 2005;62:758-765.

19. Carr DB, Gray S, Baty J, Morris JC. The value of informant vs. Individuals complaints of memory impairment in early dementia. Neurology 2000;55:1724-1726.

20. Morris JC. The Clinical Dementia Rating (CDR): current version and scoring rules. Neurology 1993;43:2412-2414.

21. Morris JC, Storandt M, Miller JP, et al. Mild cognitive impairment represents early-stage Alzheimer disease. Arch Neurol 2001;58:397-405.

22. Petersen RC, Doody R, Kurz A, et al. Current concepts in mild cognitive impairment. Arch Neurol 2001;58:19851992.

23. Blessed G, Tomlinson BE, Roth M. The association between quantitative measures of dementia and of senile change in the cerebral grey matter of elderly subjects. $\mathrm{Br}$ J Psychiatry 1968;114:797-811.

24. McKeel DW Jr, Ball MJ, Price JL, et al. Interlaboratory histopathologic assessment of Alzheimer's neuropathology: Different methodologies yield comparable diagnostic results. Alzheimer Dis Assoc Disord 1993;7:136-151.
25. Braak H, Alafuzoff I, Arzberger T, Kretzschmar H, Del Tredici K. Staging of Alzheimer disease-associated neurofibrillary pathology using paraffin sections and immunocytochemistry. Acta Neuropathol 2006;112:389-404.

26. National Institute on Aging, Reagan Institute Working Group on Diagnostic Criteria for the Neuropathological Assessment of Alzheimer's Disease. Consensus recommendations for the postmortem diagnosis of Alzheimer's disease. Neurobiol Aging 1997;18 (S4): S1-S2.

27. Fidell LS, Tabachnick BGA. California State. Preparatory data analysis. Vol. xxiii. New York, NY: US, Inc.: 2003; 711:141.

28. Comrey AL, Lee HB. A first course in factor analysis (2nd ed.). 1992.

29. Nunnally JC, Bernstein IH. Psychometric Theory, 3rd ed. New York: McGraw-Hill, 1994.

30. Osborne JW, Costello AB. Sample size and subject to item ratio in principal components analysis. Practical Assessment Research Evaluation 2004;9.

31. Welleford EA, Harkins SW, Taylor JR. Personality change in dementia of the Alzheimer's type: relations to caregiver personality and burden. Exp Aging Res 1995;21: 295-314.

32. Barber R, Snowden JS, Craufurd D. Frontotemporal dementia and Alzheimer's disease: retrospective differentiation using information from informants. J Neurol Neurosurg Psychiatry 1995;59:61-70.

33. Mychack P, Rosen H, Miller BL. Novel applications of social-personality measures to the study of dementia. Neurocase 2001;7:131-143.

34. Golden Z, Golden CJ. The differential impacts of Alzheimer's dementia, head injury and stroke on personality dysfunction. Int J Neurosci 2003;113:869-878.

35. Costa Jr PT, McCrae RR.. NEO-PIR: Professional manual. Odessa, FL: Psychological Assessment Resources, 1992.

36. Chatterjee A, Strauss ME, Smyth KA, Whitehouse PJ. Personality changes in Alzheimer's disease. Arch Neurol 1992;49:486-491.

37. Glosser G, Clark C, Freundlich B, Kliner-Krenzel L., Flaherty P, Stern M.. A controlled investigation of current and premorbid personality: Characteristics of Parkinson's disease patients. Mov Disord 1995;10:201-206.

38. Del Ser T, McKeith I, Anand R, Cicin-Sain A, Ferrara R, Spiegel R. Dementia with Lewy bodies: findings from an international multicentre study. Int J Geriatr Psychiatry 2000;15:1034-10.

39. Engelborghs S, Maertens K, Nagels G, et al. Neuropsychiatric symptoms of dementia: cross-sectional analysis from a prospective, longitudinal Belgian study. Int J Geriatr Psychiatry 2005;20:1028-1037.

40. Moran M, Walsh C, Lynch A, Coen RF, Coakley D, Lawlor BA. Syndromes of behavioral and psychological symptoms in mild Alzheimer's disease. Int J Geriatr Psychiatry 2004;19:359-364.

41. Cummings JL.. Fluctuations in cognitive function in dementia with Lewy bodies. Lancet Neurol 2004;3:266. 